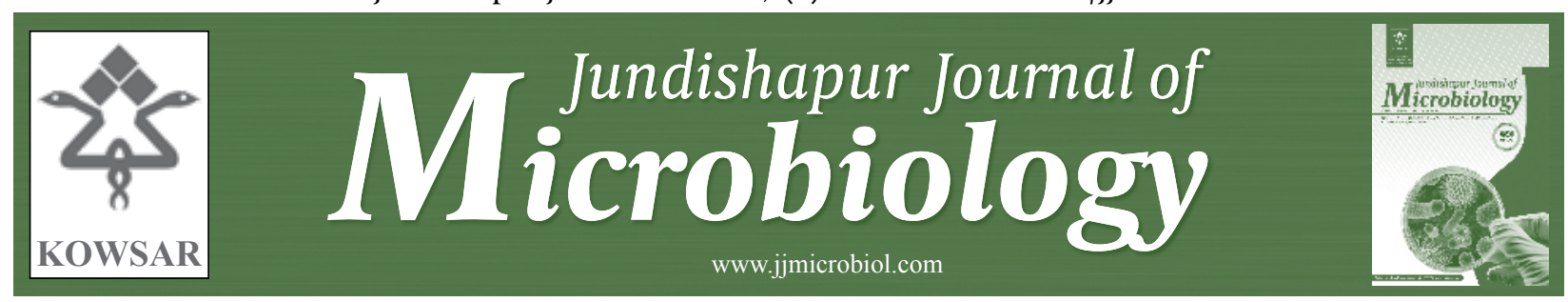

\title{
A Review of Epidemiology, Diagnosis and Management of Brucellosis for General Physicians Working in the Iranian Health Network
}

\author{
Seyed Mohammad Alavi ${ }^{{ }^{*}}$, Mohammad Esmaeil Motlagh ${ }^{2}$ \\ ${ }^{1}$ Jundishapur Infectious and Tropical Diseases Research Center, Department of Infectious Diseases, Razi Hospital, Ahvaz Jundishapur University of Medi- \\ cal Sciences, Ahvaz, IR Iran \\ ${ }^{2}$ Department of Community Medicine, Ahvaz Jundishapur University of Medical Sciences, Ahvaz, IR Iran
}

\begin{tabular}{|c|c|}
\hline A R T I C L E I N F O & A B S T R A C T \\
\hline Article type: & Although rare in industrialized countries, brucellosis continues to be a major public \\
\hline Review Article & health problem in developing countries such as Iran. General physicians (GP) as well as \\
\hline Article history: & lem as well as an occupational hazard. The aim of this article is to review the informa- \\
\hline Received: 03 May 2011 & tion about the epidemiology, immunopathogenesis, diagnosis and occupational risks \\
\hline Revised: 20 Sep 2011 & associated with the prevention and treatment of brucellosis. Information obtained from \\
\hline Accepted: Oct 2011 & previous investigations on brucellosis has yielded better knowledge about this illness. \\
\hline $\begin{array}{l}\text { Keywords: } \\
\text { Brucellosis }\end{array}$ & $\begin{array}{l}\text { consultations, early diagnosis and treatment to attending people or patients at health } \\
\text { care units. }\end{array}$ \\
\hline
\end{tabular}

Occupational Exposure

Health Personnel

Copyright $\odot 2012$ Kowsar Corp. All rights reserved.

- Implication for health policy/practice/research/medical education:

This article is a useful guide for general physician involved in public health to manage infectious diseases.

- Please cite this paper as:

Alavi SM, Motlagh ME. A Review of Epidemiology, Diagnosis and Management of Brucellosis for General Physicians Working in the Iranian Health Network. Jundishapour J Microbiol. 5(2):384-7. DOI: 10.5812/jjm.3248

\section{Introduction}

Brucellosis is a common infectious disease in both human and animals which affects human populations worldwide (1). Although seldom seen in developed countries, brucellosis continues to be a major public health problem in countries such as Iran $(2,3)$. Health care workers are exposed to a number of occupational hazards including infectious agents (4). Brucellosis is an occupational disease in people engaged with Brucella species such as laboratory personnel, biology researchers and veterinarians involved with animal brucellosis control programs (1-4). Ministry of Health, medical science uni-

* Corresponding author: Seyed Mohammad Alavi, Jundishapur Infectious and Tropical Diseases Research Center, Ahvaz ,Jundishapur University of Medical Sciences, Ahvaz, IR Iran. Tel: +98-6113387724, Fax: +98-611333651; Email:alavi.seyedmohammad@yahoo.com

DOI: $10.5812 /$ jjm.3248

Copyright $\odot 2012$ Kowsar Corp. All rights reserved. versities and health care providers, who are concerned with public health, should not overlook the dangers of infectious pathogen exposure in health care settings (4). The aim of this article is to review the current information about brucellosis such as epidemiology in the community and in particular hosts, pathogenesis, and occupational risks associated in the health care workplace. A systematic review of the literature on the epidemiology, diagnosis, treatment and prevention of brucellosis from 1991 to 2010 using computerized bibliographic databases which include Pub Med, Current Content, Scopus, EMBASE and Iran Medex was carried out to increase understanding of brucellosis in health care settings.

\section{Primary Health Centre}

The Primary Health Centre (PHC) is the fundamental unit of the public health system providing services throughout Iran, from remote mountain areas to in- 
ner urban areas in the country's capital (5). The Iranian Health Care Network provides health services through first line health services called in Iran Khanehe Behdasht, health centers and tertiary hospitals $(5,6)$. Since 1984 , the activities of the health system have resulted in a dramatic decrease in the burden of common and endemic infectious diseases (6).

\section{Epidemiology}

Brucellosis exists in most parts of Iran especially in the villages and nomadic areas where livestock are usually kept in close human proximity (7-9). The disease is produced by several different Brucella species, of which four, namely, Brucella melitensis, Brucella abortus, Brucella suis and Brucella canis are capable of causing human infections (2). The most prevalent livestock is the goat (9), so B. melitensis is the most common cause of human brucellosis in this area $(3,7,10)$. Routes of transmission from animal to human include: 1 - direct contact with infected animals, 2- inhalation of contaminated aerosols, and 3ingestion of unpasteurized dairy products (2). Although risk factors for the acquisition of infection among health care workers as well as the general population includes the consumption of fresh cheese, contact with animal skins and eating undercooked meat or raw milk, brucellosis is also an occupational hazard for certain jobs in health settings such as veterinarian and laboratory personnel (11). Human brucellosis has been accidentally induced among health workers providing animal vaccines as a result of exposure to those vaccines (2). Personto-person transmission of brucellosis is unusual; however, blood transfusions and bone marrow transplants are known sources of brucellosis, emphasizing the need for antibody detection, especially in endemic areas (2). There are some reports of more than one case of brucellosis in a household; therefore, antibody investigation of index case's contacts is recommended.

\section{Pathogenesis and Immunity}

Pathogenesis of Brucella is dependent on the species, route of transmission, size of the infectious inoculums, nutritional and immune status of the host. The brucellae are intracellular pathogens which multiply within the phagocyte of the host. Brucellae within macrophages become localized in organs of the reticuloendothelial system, such as the lymph nodes, liver, spleen, and bone marrow (10). The eventual elimination of virulent brucellae depends on the activation of macrophages with the development of Th I-type cell-mediated immunity. AntiBrucella activity of macrophages include tumor necrosis factor-a, tumor necrosis factor-y, interleukin-I, and interleukin-12. Haghirizadeh et al. reported that chronic and relapsed brucellosis are associated with diminished values in interleukin-12 (12). The appearance of Ig M-antibodies within the first week of infection is a clue to the immune response against brucellosis which switches to IgG after the second week. Antibody titers decline slowly after treatment or recovery. Persistent high titers of IgG is a laboratory predictor of relapse or chronic infection (2).

\section{Clinical Manifestations}

\subsection{Signs and Symptoms}

Clinical findings of brucellosis such as fever, headache, back pain, sweats, malaise, and anorexia are usually nonspecific. The onset of clinical manifestations can be insidious or acute, beginning within 2 to 4 weeks following infection. Compared with the symptoms, there are often few signs in the physical examination (13). Mild lymphadenopathy and splenomegaly or hepatomegaly may be seen in a portion of patients (1). Recurrence of symptoms after therapy may or may not be associated with relapse of the disease. Bacteriologic relapse usually appears within 3 to 6 months after discontinuing drug treatment and is not usually caused by antibiotic resistance (14). In chronic brucellosis, symptoms can recur after a long period of time and are associated with fever which is one of the most objective signs of infection. An important laboratory finding is the persistence of high titers of IgG antibodies (1). In some patients persistent nonspecific symptoms may be seen without elevated titers of IgG. The reason for this condition is not clear, but some investigators believe that it may be due to exacerbation of preexisting psychoneurosis by the infection (15).

\subsection{Complications}

In some cases patients with brucellosis present with a range of complications. The most important complications of brucellosis (2) are as follows; gastrointestinal symptoms - anorexia, nausea, vomiting, pain, diarrhea, and constipation, which are observed in $70 \%$ of brucellosis cases. Hepatobiliary system; hepatic involvement is common in brucellosis. Skeletal complications; osteoarticular complications are the most common focal forms of the disease and have been reported in $10 \%$ to $80 \%$ of cases depending on the series, the ages of the patients, and the infecting Brucella spp. Nervous system; depression and lack of concentration are common symptoms in brucellosis, however direct invasion of the central nervous system occurs in less than $5 \%$ of cases. Neurological syndromes in brucellosis include; meningitis, encephalitis, myelitis-radiculoneuronitis, brain abscess, epidural abscess, granuloma, and demyelization and meningovascular syndromes (2).

Cardiovascular involvement; endocarditis occurs in less than $2 \%$ of cases, but it accounts for the majority of brucellosis-related deaths. Genitourinary complications; interstitial nephritis, pyelonephritis, glomerulonephritis, and immunoglobulin nephropathy have been reported. Epididymo-orchitis occurs in up to $10 \%$ of men with brucellosis (16). Hematological complications; hematologic 
manifestations of brucellosis include anemia, leukopenia, thrombocytopenia, and clotting disorders. Ocular complications; uveitis is generally a late complication, consisting variably of chronic iridocyclitis, nummular keratitis, multi focal choroiditis, and optic neuritis (17).

\section{Diagnosis}

Diagnosis of brucellosis is based on clinical findings (nonspecific), history (including occupation, travel to an endemic area and ingestion of unpasteurized dairy products) and laboratory tests such as serology or bacterial isolation. Although modern diagnostic techniques such as nucleic acid amplification have been introduced, they are not yet widely available especially in areas with restricted resources. Blood and tissue based polymerase chain reactions (PCR) can detect brucellosis, although PCR is more sensitive and quicker than blood culture (16).

\subsection{Definite Diagnosis}

A definite diagnosis requires the isolation of brucellae from the blood, bone marrow or other tissues (2). However, cultural examinations are time consuming, hazardous and not sensitive. Thus, clinicians often rely on indirect proof of infection.

\subsection{Presumptive Diagnosis}

A variety of serological tests have been developed, but at least two serological tests should be combined to confirm an active infection. Usually, the standard tube agglutination (STA) test (Wright) is used first and the 2mercaptoetanole (2ME) test will confirm its results (with 97.1\% sensitivity and $100 \%$ specificity) $(11,13)$. In the absence of a bacteriologic examination an initial diagnosis is made by showing high titers of IgG- antibodies against Brucella in the serum. $(13,14)$. According to the National Program against Brucellosis (NPB) diagnosis is based on serological tests (Wright $>1 / 80$ and $2 \mathrm{ME}>1 / 20$ ) in the presence of clinical findings suggestive of brucellosis (3, 7). Other techniques such as polymerase chain reaction (PCR) have been used to diagnose brucellosis (2). Khosravi et al. reported that the high degree of sensitivity of a PCR assay, together with its speed, versatility in sample handling, and risk reduction for laboratory personnel, make this technique a very useful tool for the diagnosis of brucellosis, compared with conventional culture method (10).

\subsection{Diagnostic Clinical Criteria}

Alavi et al. in their work showed that in limited resource areas where laboratory facilities are not available clinical criteria are as effective as the Iranian NPB guidelines (3) in the diagnosis of brucellosis. These clinical criteria include; three major, or one major and three minor, or five minor. Major criteria were close animal contact, fever and joint involvement. Minor criteria were sweating, headache, weight loss, chills and malaise (13).

\section{Treatment}

\subsection{Standard Treatment}

Using antibiotics against Brucella relieves symptoms, lowers the duration of the disease and decreases the risk of relapse or complications. A regimen of doxycycline (100 mg every 12 hours PO for 6 weeks) plus streptomycin (I g/day $1 \mathrm{M}$ for 2 to 3 weeks) is the treatment of choice (2, 3).

\subsection{Oral Treatment}

A combination of doxycycline $(200 \mathrm{mg} /$ day PO for 6 weeks) plus rifampin (600 to $900 \mathrm{mg} /$ day PO for 6 weeks) has the advantage of being a total oral regimen, but it is not recommended in tuberculous areas or in complicated cases such as spondylitis $(17,18)$.

\subsection{Selection an Antibiotic Regimen}

Selection of an antibiotic regimen as the first choice and duration of chemotherapy should be based on the location of the disease and the underlying conditions (19). The World Health Organization (WHO) has recommended doxycycline plus rifampicin or doxycycline plus streptomycin as combination therapies for the treatment of brucellosis. Good results have been achieved with these regimens, however the reported relapse rates of brucellosis are still as high as $14.4 \%$.

The most effective and the least toxic chemotherapy for human brucellosis are still undetermined (20). In countries with a high prevalence of tuberculosis and brucellosis such as Iran, frequent usage of rifampicin (not combined with other anti TB drugs) in the treatment for brucellosis can result in resistant Mycobacterium tuberculosis. Alavi et al. in their study conducted on nomads in Khuzestan showed that a co-trimoxazole plus doxycycline regimen has a better therapeutic effect than a doxycycline plus rifampicin regimen (21). Due to the lack of medical facilities such as safe injection and expert health care workers in remote areas in which nomads are living, this regimen compared with standard treatment has the advantage of being administered orally. Other regimens such as co trimoxazole-rifampicin have been associated with high rates of treatment failure (22).

\section{Prevention}

\subsection{Eradication of Brucella in Animals}

Prevention of human brucellosis is based on the eradication of animal brucellosis through testing, while a slaughter strategy has been used successfully in countries which are now free of brucellosis. Effective live bacterial vaccines for animals against B. abortus and B. melitensis exist. There are no recommendations for a vaccine to prevent human brucellosis, and there is controversy around Brucella postexposure chemoprophylaxis $(17,18,23,24)$. 


\section{Recommendations}

Here are some recommendations to be considered for brucellosis prevention: 1-Conduct public awareness and education campaigns. 2- Wear rubber gloves while handling animals, meat, blood and viscera suspected to be infected with Brucella. Wear bandages or dressings to protect wounds from direct exposure. 3- Pasteurize all milk and other dairy products, especially cheese. Cheese aged less than 3 months may still be contaminated. Unpasteurized dairy products should not be eaten while traveling to endemic areas. 4- Control brucellosis in animals through immunization, surveillance and screening of all animals in endemic areas. There are live vaccines for B. abortus and B. melitensis, but they are not recommended for human use as they can cause infection. Immunity to brucellosis after an infection lasts only about two years. 5- Slaughter infected animals such as cattle, goats and sheep. Avoid unsafe contact with animals suspected to be infected with Brucella species (25).

\section{Acknowledgement}

None Declared.

\section{Financial Disclosure}

None Declared.

\section{Funding/Support}

None Declared.

\section{References}

1. Nimri LF. Diagnosis of recent and relapsed cases of human brucellosis by PCR assay. BMC Infect Dis. 2003;3:5.

2. Young EJ. Brucella species. In: Mandell GL, Douglas RG, Bennett JE, editors. Principles and practice of infectious diseases. Churchill Livingstone: Wiley; 2005. p. 2669-74.

3. Ghasemi R. The most important control activities in Iran. [cited]; Available from: http://www. elib. ir/persian/public_health_ ebook/09_16. htm.

4. Moore RM, Jr. , Kaczmarek RG. Occupational hazards to health care workers: diverse, ill-defined, and not fully appreciated. Am J Infect Control.1990;18 (5):316-27.

5. Wikipedia the free encyclopedia. Lean. 2011 [updated 20112011 Sep24; cited]; Available from: http://en. wikipedia. org/wiki/Lean.

6. Asadi-Lari M, Sayyari AA, Akbari ME, Gray D. Public health improvement in Iran-lessons from the last 20 years. Public Health. 2004;118 (6):395-402.

7. Tabatabaei S, Zahraei M, Ahmadnia H, Ghotbi M, Rahimi F. Princi- ples of disease prevention and Surveillance. Center for diseases control, Vice-chancellor for health, ministry of health and medical education, 1st ed. 2008.

8. Alavi SM, Rafiei A, Nikkhooi A. The effect of lifestyle on brucellosis among nomads in Khuzestan province of Iran. PakJ Med Sci. 2007;23 (3):358-60.

9. Alavi SM RA, Nikkhoi. Seroepidemiological survey among nomads in Khuzestan, Iran. Infect Dis. 2006;33:345-50.

10. Khosravi AD, Abassi E, Alavi SM. Isolation of Brucella melitensis and Brucella abortus from brucellosis patients by conventional culture method and polymerase chain reaction technique. PakJ Med Sci. 2006;22 (4):396-400.

11. Alavi SM, Alavi SMR, Alavi L. Relapsed human brucellosis and related risk factors. PakJ Med Sci. 2009;25 (1):46-50.

12. Haghirizadeh Roudan M EM, Alavi SM, Pezeshki M. A study on cytokines production in patients with brucellosis. Sci Med JAJUMS. 2002;34:20-4

13. Alavi SM, Alavi L. Comparative study of current diagnostic method with clinical based method for brucellosis: presentation of diagnostic clinical criteria in limited resource area. Jundishapur J Microbiol. 2011;3 (3):121-4.

14. Ariza J, Bosch J, Gudiol F, Linares J, Viladrich PF, Martin R. Relevance of in vitro antimicrobial susceptibility of Brucella melitensis to relapse rate in human brucellosis. Antimicrob Agents Chemother. 1986;30 (6):958-60.

15. Cluff LE. Medical aspects of delayed convalescence. Rev Infect Dis. 1991;13 (Suppl 1):S138-40.

16. Kasper DL, Braunwald E, Fauci AS, Hauser SL, Longo DL, Jameson JL, et al. Harrisons principles of internal medicine 16th ed. 2005.

17. Ariza J, Bosilkovski M, Cascio A, Colmenero JD, Corbel MJ, Falagas ME, et al. Perspectives for the treatment of brucellosis in the 21st century: the Ioannina recommendations. PLoS Med. 2007;4 (12):e317.

18. Skalsky K, Yahav D, Bishara J, Pitlik S, Leibovici L, Paul M. Treatment of human brucellosis: systematic review and meta-analysis of randomised controlled trials. BMJ. 2008;336 (7646):701-4.

19. Solera J. Treatment of human brucellosis. J Med Liban. 2000;48 (4):255-63.

20. Karabay O, Sencan I, Kayas D, Sahin I. Ofloxacin plus rifampicin versus doxycycline plus rifampicin in the treatment of brucellosis: a randomized clinical trial [ISRCTN11871179]. BMC Infect Dis. 2004;4:18.

21. Alavi S, Rajabzadeh A. Comparison of two chemotherapy regimen: Doxycycline-rifampicin and doxycycline cotrimoxazol in the brucellosis patients Ahwaz, Iran, 2004-2006. Pakistan J Med Sci. 2007;23 (6):889.

22. Roushan MR, Gangi SM, Ahmadi SA. Comparison of the efficacy of two months of treatment with co-trimoxazole plus doxycycline vs. co-trimoxazole plus rifampin in brucellosis. Swiss Med Wkly. 2004;134 (37-38):564-8.

23. Robichaud S, Libman M, Behr M, Rubin E. Prevention of laboratory-acquired brucellosis. Clin Infect Dis. 2004;38 (12):e119-22.

24. Maley MW, Kociuba K, Chan RC. Prevention of laboratory-acquired brucellosis: significant side effects of prophylaxis. Clin Infect Dis. 2006;42 (3):433.

25. Taleski V, Zerva L, Kantardjiew T, Cevetnic Z, Erski-Biljic M, Nikolovski B. An overview of the epidemiology and epizoology of brucellosis in selected countries of central and south east Europe. Vet Microbiol. 2002;90 (1-4):147-55. 